\title{
Effect of Sitagliptin, Pioglitazone and Dapagliflozine on Myocardial Infarction Induced Experimentally in Diabetic Rats
}

\author{
Ahmed F. Bahriz, Yassmin M. Ismaiel, Abeer A. Abdelhameed, Fatma A. Elsayed
}

\begin{abstract}
Department of Clinical Pharmacology, Benha faculty of medicine, Benha University, Egypt.
\end{abstract}

Correspondence to: Fatma A. Elsayed, Department of Clinical Pharmacology, Benha faculty of medicine, Benha University, Egypt.

Email:

fatmaabdelmoaty2021@gmail. com

Received: 18 January 2021

Accepted: 28 January 2021

\section{Abstract:}

Background: Diabetes is associated with both micro- and macrovascular complications. Aim of the study: The aim of the present study is to evaluate the prophylactic effect of Sitagliptin, Pioglitazone and Dapagliflozine on Isoprenaline (ISO) induced myocardial infarction in type II diabetic rats, regarding these parameters: lipid profile, blood glucose, kidney function, troponin, MDA, tumor necrosis factor- $\alpha$, HR, ST elevation and histopathological changes of myocardium. Methods: Rats were classified into: Group I: control normal group. Group II: was not treated diabetic (diseased group). Group III: was treated with sitagliptin. Group IV: was treated with pioglitazone. Group V: was treated with dapagliflozine. Treated groups received drugs for 4 weeks. Results: Treated groups showed significant improvement in all parameters and improvement of the histopathology of the myocardium. A significant improvement in the parameters was seen in the treated groups at the end of the $4^{\text {th }}$ week. Conclusion: Our study revealed that dapagliflozine produced more improvement in blood glucose level, and there was no significant difference between it and pioglitazone in improving lipid profile. Pioglitazone was superior in decreasing creatinine level, serum troponin, HR, ST elevation, MDA, TNF $\alpha$ and histopathological changes of myocardium. There was no significant difference between the three drugs regarding to their effect on blood urea. So our drugs ,mainly pioglitazone may have a prophylactic effect against MI in diabetic rats, this may be due to their antioxidant and anti-inflammatory effect through reduction of MDA and TNF $\alpha$ respectively, glycemic control and improvement of dyslipidemia.

Key words: diabetes mellitus, myocardial infarction, sitagliptin, pioglitazone, dapagliflozine. 

Abbreviations: GLP-1; glucagon-like peptide-1, DPP-IV; dipeptidyl peptidase IV, SGLT2; sodiumglucose cotransporter-2 ISO; Isoprenaline, MI; Myocardial infarction, MDA; malondialdehyde, $\mathrm{TNF} \alpha$; tumor necrosis factor $\alpha, \mathrm{HR}$; heart rate.

\section{Introduction}

The American Diabetes Association (ADA) defined diabetes mellitus (DM) as a group of metabolic diseases characterized by hyperglycemia resulting from defects in insulin secretion, insulin action or both. The chronic hyperglycemia of diabetes is associated with long-term damage, dysfunction and failure of different organs, especially the eyes, kidneys, nerves, heart and blood vessels [1].

Myocardial ischemia represents a condition of sufferance for cardiomyocytes due to coronary blood flow reduction as compared to their metabolic needs, and it may exhibit through several clinical conditions [2].

People with type 2 diabetes mellitus are at increased risk of cardiovascular disease, heart failure and death, as compared with the general population. Studies show that the excess risks associated with diabetes mellitus are mediated primarily by hyperglycemia [3] and overall poor risk factor control. Effective treatment of traditional cardiovascular risk factors has reduced the excess risk of atherosclerotic cardiovascular disease (CVD), such as acute myocardial infarction (AMI) and stroke in people with type 2 diabetes mellitus.

In the last two decades, several studies have demonstrated reductions in the risk of cardiovascular outcomes and mortality in patients with T2DM with improved glucose and cholesterol-lowering therapies [4]. Nevertheless, macrovascular disease remains the most common cause of death in T2DM patients and new diabetes therapies are highly desired, especially if they can offer cardiovascular benefits [5].

Sitagliptin is a potent and highly selective DPP-4 competitive inhibitor that does not affect the closely related enzymes DPP-8 or DPP-9 at therapeutic concentrations [6]. Sitagliptin found to enhance circulating glucagon-like peptide-1 (GLP-1) levels through inhibition of dipeptidyl peptidase IV (DPP-IV) activity [7] which, in turn, provides cardiovascular protection probably through the anti-inflammatory and anti-atherosclerotic activities of GLP-1[8]. Reduces blood glucose levels, in either the postprandial or the fasting state [9].

Multiple studies have suggested that pioglitazone, a peroxisome proliferatoractivated receptor $\gamma(\operatorname{PPAR} \gamma)$ agonist, used as an insulin-sensitizing agent in the 
treatment of type 2 diabetes mellitus (T2DM), may have anti-atherosclerotic effects [10]. Both insulin resistance and systemic low-grade inflammation are associated with atherosclerotic plaque formation and pioglitazone improves insulin resistance and reduces systemic inflammation [11].

Dapagliflozine is a highly potent and reversible SGLT2 inhibitor that is > 1400 times more selective for SGLT2 than SGLT1, the main transporter responsible for glucose absorption in the gut [12]. The mechanism of action of dapagliflozine influences a number of CVD risk factors, in particular, decreasing blood pressure, reducing body weight (predominantly through reductions in total body fat mass, including visceral adipose tissue), reducing waist circumference, and lowering albuminuria and serum uric acid levels, with allow intrinsic risk of hypoglycemia [13].

Isoprenaline is a potent $\beta$-adrenergic agonist, increases the myocardial oxygen demand by mixture of its positive inotropic and chronotropic actions. Administration of isoprenaline in high doses to animals produces infarct like lesions in the heart similar to those present in MI in humans [14].

\section{Materials and Methods}

\section{Animals:}

It is a prospective study carried out of (40) adult male albino rats obtained from (Experimental Animal Breeding Farm, Helwan-Cairo) weighing between 150- 200 $\mathrm{g}$ (at the beginning of the study), were used for in-vivo experiments. They were acclimatized for one week and were caged (8 rat/cage) in fully ventilated room at room temperature in the Pharmacology Department, Benha Faculty of Medicine. The study was carried out from $1^{\text {st }}$ of September 2019 to $15^{\text {th }}$ of December 2019. Rats were fed a standard chow with water. This study was approved from ethical committee of Benha Faculty of Medicine.

\section{Drugs}

D-Fructose (El-Nasr pharmaceuticals Chemicals Co. ADWIC, Egypt), Streptozotocine (Sigma Aldrich), Isoprenaline (Sigma CO., USA), Formalin solution (neutral 10\% formalin) (ElGomhoria Pharmaceutical Chemical Co., Egypt), Dapagliflozine hemihydrate (Janssen, USA). Pioglitazone hydrochloride (Unipharma., Egypt), Sitagliptin (Novartis, USA), Urethane (Ethyl carbamate): (Prolabo, Paris), Heparin ampoule (Novo Industry, Demark). 


\section{Experimental groups and procedures:}

The rats were classified into 5 equal groups $(\mathrm{n}=6)$ as follow;

Control group (G1); formed of normal animals. They were allowed standard normal diet and water. They received no drugs. Diseased group (G2); Non-treated diabetic rats; diabetes was induced by administration of $20 \%$ fructose solution in drinking water for 2 weeks, then intra peritoneal injection of a low dose STZ (40 $\mathrm{mg} / \mathrm{kg}$ b.w.), was done $[15,16]$.

Sitagliptin treated diabetic group (G3); sitagliptin was administered by gastric gavage in a dose $10 \mathrm{mg} / \mathrm{kg} /$ day for 4 weeks [17]. Pioglitazone treated diabetic group (G4); Pioglitazone was administered by gastric gavage in a dose $10 \mathrm{mg} / \mathrm{kg} /$ day for 4 weeks [18]. Dapagliflozine treated diabetic group (G5); Dapagliflozine was administered by gastric gavage in a dose $1 \mathrm{mg} / \mathrm{kg} /$ day for 4 weeks [19].

The treated groups received the drugs for 4 weeks then myocardial infarction was induced in all diabetic groups by injection of isoprenaline subcutaneously (S.C) in the abdominal region $(150 \mathrm{mg} / \mathrm{kg})$ dissolved in $2 \mathrm{ml}$ saline. It was injected as a single dose [20]. At the end of the experiment, the animals were anesthetized with Urethane at dose $1.25 \mathrm{~g} / \mathrm{kg}$ body weight, half injected intraperitoneally for rapid action and the other half was injected subcutaneously for slow sustain action[21], then ECG was done $2 \mathrm{hrs}$ after injection of ISO.

After ECG recording, the chest was then rapidly opened and blood sample about $(3 \mathrm{ml})$ was collected from the right ventricle before removal of the heart from chest. Blood samples were incubated at $37^{\circ} \mathrm{C}$ until clotting, and then centrifuged at 3000 revolution per minute (rpm) for 15 minutes, for separation of serum and stored at $-20^{\circ} \mathrm{C}$ for biochemical analysis of cardiac troponin, blood glucose, lipid profile and kidney function tests. The heart was divided into two halves.

Half was immediately washed with normal saline, kept in liquid nitrogen, and stored at $-20^{\circ} \mathrm{C}$ for MDA and TNF $\alpha$ assays. The other half was kept in formaldehyde to be stained with Hematoxylin and Eosin.

\section{ECG Monitoring}

The anesthetized rats were placed in the supine position on a board and ECG was traced continuously by means of needle electrodes. These electrodes were inserted subcutaneously into the paw pads of the rat and connected to ECG device. For each rat, Lead II was recorded being the most 
informative one (right forelimb to left hindlimb). ECG tracing was analyzed for Q, R, $\mathrm{T}$ waves and heart rates.

\section{Measurement of blood glucose:}

By GOD-PAP enzymatic colorimetric method; Glucose is determined after enzymatic oxidation in the presence of glucose oxidase (GOD). The formed hydrogen peroxide reacts under catalysis of peroxidase (POD) with phenol and 4aminoantipyrine (PAP) to form a red violet quinoneimine dye as indicator.[22]

\section{Measurement of Serum Troponin I activity:-}

Serum Troponin-I activity was determined according to the method done by Bodor 1994 [23].

\section{Cardiac malondialdehyde (MDA) assay:}

Portions of the myocardium were homogenized in a saline solution $(0.9 \%)$ and centrifuged at $3000 \mathrm{rpm}$ for $15 \mathrm{~min}$; the supernatant was kept at $-20^{\circ} \mathrm{C}$. The antioxidant MDA kit (Cambridge, UK, Cat. No. ab118970) was used to assess the cardiac content according to the enzymatic colorimetric assay method [24]. .

\section{Cardiac TNF $\alpha$ assay:}

Cardiac TNF- $\alpha$ was measured by ELISA [25].

\section{Measurement of serum lipid profile:}

- Cholesterol (TC) and triglycerides (TG): This was carried out by "Enzymatic colorimetric test",[26,27] Respectively, HDL-cholesterol: This is carried out by "separation of high-density lipoproteins (HDL) and determination of cholesterol bound to these fractions " [28]. LDLcholesterol $=$ Total Cholesterol-(HDLcholesterol+ TG/5) (mg/dl) [29].

Measurement of serum urea and creatinine:

This was carried out by "Enzymatic colorimetric test" [30, 31].

\section{Histopathological examination of the cardiac tissue:}

Transverse sections ( $2 \mathrm{~mm}$ thickness) of the left ventricle free wall at the papillary muscle level, were stained with hematoxylin and eosin then cardiac sections were examined for the presence of myocyte degenerative changes and infarction like necrosis [32] .

\section{Statistical analysis:}

The collected data were summarized in terms of mean \pm Standard Deviation (SD). Comparisons between the different study groups were carried out using the one-way analysis of variance (ANOVA) followed by 
post hoc tests using the LSD method using the Statistical Package for Social Science (SPSS) program, version 19 (Chicago IL USA, 2000). $P$ value $<0.05$ was considered statistically significant.

\section{Results}

Induction of DM by HF-STZ (high fructose+streptozotocin) resulted in significant increase in blood glucose, urea, creatinine, total cholesterol, LDL, triglycerides and significant decrease in HDL levels.

Regarding, the treated groups there was significant improvement in blood glucose, urea, creatinine, total cholesterol, LDL, triglycerides and significant increase in HDL level. (table 1). Dapagliflozine produced more improvement in bl. glucose, cholesterol and HDL levels, while pioglitazone produced more improvement in LDL, TG, urea and creatinine levels, but the difference between dapagliflozine and pioglitazone was non-significant in most of these parameters.

Induction of MI by isoprenaline resulted in significant increase in HR, ST segment elevation, serum troponin, MDA and TNF $\alpha$. Also there was significant increase in histopathological cardiac injury score. Regarding, the treated groups there was significant improvement in HR, ST segment elevation, serum troponin, MDA and TNF $\alpha$. (table 2-figures 1, 2,3, 4 \&5)

There was a significant improvement in histopathological cardiac injury score compared to diseased group. (Figures 6, 7, $8,9 \& 10)$

Although the three drugs showed improvement in HR, ST segment elevation, serum troponin, MDA, TNFa and histopathological cardiac injury score. Pioglitazone produced more improvement in all of these parameters. 
Table (1): The effect of treatment with sitagliptin, pioglitazone and dapagliflozine on (blood glucose,urea, creatinine, total cholesterol, LDL, triglycerides and HDL) on experimentally induced diabetes in rats

\begin{tabular}{|c|c|c|c|c|c|c|c|c|c|c|}
\hline & \multicolumn{2}{|c|}{ Control group } & \multicolumn{2}{|c|}{$\begin{array}{l}\text { Diseased } \\
\text { group }\end{array}$} & \multicolumn{2}{|c|}{ sitagliptin group } & \multicolumn{2}{|c|}{$\begin{array}{l}\text { Pioglitazone } \\
\text { group }\end{array}$} & \multicolumn{2}{|c|}{$\begin{array}{l}\text { dapagliflozine } \\
\text { group }\end{array}$} \\
\hline & Mean & $\pm \mathrm{SD}$ & Mean & $\pm \mathrm{SD}$ & Mean & $\pm \mathrm{SD}$ & Mean & $\pm \mathrm{SD}$ & Mean & $\pm \mathrm{SD}$ \\
\hline $\begin{array}{l}\text { Bl.glucose } \\
\text { (mg/dl) }\end{array}$ & \multicolumn{2}{|c|}{$91.33 \pm 9.07$} & \multicolumn{2}{|c|}{$\begin{array}{c}287.00 \pm 24.06 \\
\mathbf{a}\end{array}$} & \multicolumn{2}{|c|}{$\begin{array}{c}175.67 \pm 11.59 \\
\mathbf{a b}\end{array}$} & \multicolumn{2}{|c|}{$\begin{array}{c}136.33 \pm 11.50 \\
\text { abc }\end{array}$} & $123.67 \pm 1$ & \\
\hline $\begin{array}{l}\text { S.urea } \\
\text { (mg/dl) }\end{array}$ & \multicolumn{2}{|c|}{$23.00 \pm 2.00$} & \multicolumn{2}{|c|}{$\begin{array}{c}40.33 \pm 2.52 \\
\mathbf{a}\end{array}$} & \multicolumn{2}{|c|}{$\begin{array}{c}27.67 \pm 2.08 \\
\mathbf{a b}\end{array}$} & \multicolumn{2}{|c|}{$\begin{array}{c}25.67 \pm 2.08 \\
\text { b }\end{array}$} & $25.81 \pm 1$ & \\
\hline $\begin{array}{l}\text { s.creatinine } \\
\text { (mg/dl) }\end{array}$ & \multicolumn{2}{|c|}{$0.91 \pm 0.07$} & \multicolumn{2}{|c|}{$1.74 \pm 0.11$} & \multicolumn{2}{|c|}{$1.29 \pm 0.07$} & \multicolumn{2}{|c|}{$\begin{array}{c}1.13 \pm 0.06 \\
\text { abc }\end{array}$} & 1.2 & \\
\hline $\begin{array}{l}\text { Cholesterol } \\
\text { (mg/dl) }\end{array}$ & \multicolumn{2}{|c|}{$106.33 \pm 7.37$} & \multicolumn{2}{|c|}{$243.00 \pm 22.61$} & \multicolumn{2}{|c|}{$173.33 \pm 13.01$} & \multicolumn{2}{|c|}{$139.00 \pm 14.11$} & 121. & 0.82 \\
\hline LDL (mg/dl) & \multicolumn{2}{|c|}{$66.57 \pm 0.40$} & 96.67 & -2.37 & \multicolumn{2}{|c|}{$78.97 \pm 1.76$} & \multicolumn{2}{|c|}{$69.23 \pm 1.16$} & 71. & 15 \\
\hline $\begin{array}{l}\text { Triglyceride } \\
\text { (mg/dl) }\end{array}$ & \multicolumn{2}{|c|}{$129.67 \pm 12.50$} & \multicolumn{2}{|c|}{$\begin{array}{c}226.67 \pm 19.76 \\
\mathbf{a}\end{array}$} & \multicolumn{2}{|c|}{$\begin{array}{l}152.67 \pm 10.60 \\
\mathbf{a b}\end{array}$} & \multicolumn{2}{|c|}{$\begin{array}{c}133.67 \pm 12.90 \\
\text { b }\end{array}$} & \multicolumn{2}{|c|}{$\begin{array}{l}135.67 \pm 10.41 \\
\text { b }\end{array}$} \\
\hline HDL (mg/dl) & 53.3 & 2.52 & 35.67 & -2.08 & 42.6 & 3.06 & 47.00 & 3.00 & 50. & \\
\hline
\end{tabular}
(a) significant versus control (G1).
(b) Significant versus diseased group (G2).
(c) Significant versus sitagliptin group (G3).
(d) Significant versus pioglitazone group (G4). 
Table (2): The effect of treatment with sitagliptin, pioglitazone and dapagliflozine on (HR,STsegment elevation ,serum troponin ,MDA and TNF $\alpha$ ) on experimentally induced myocardial infarction in diabetic rats:

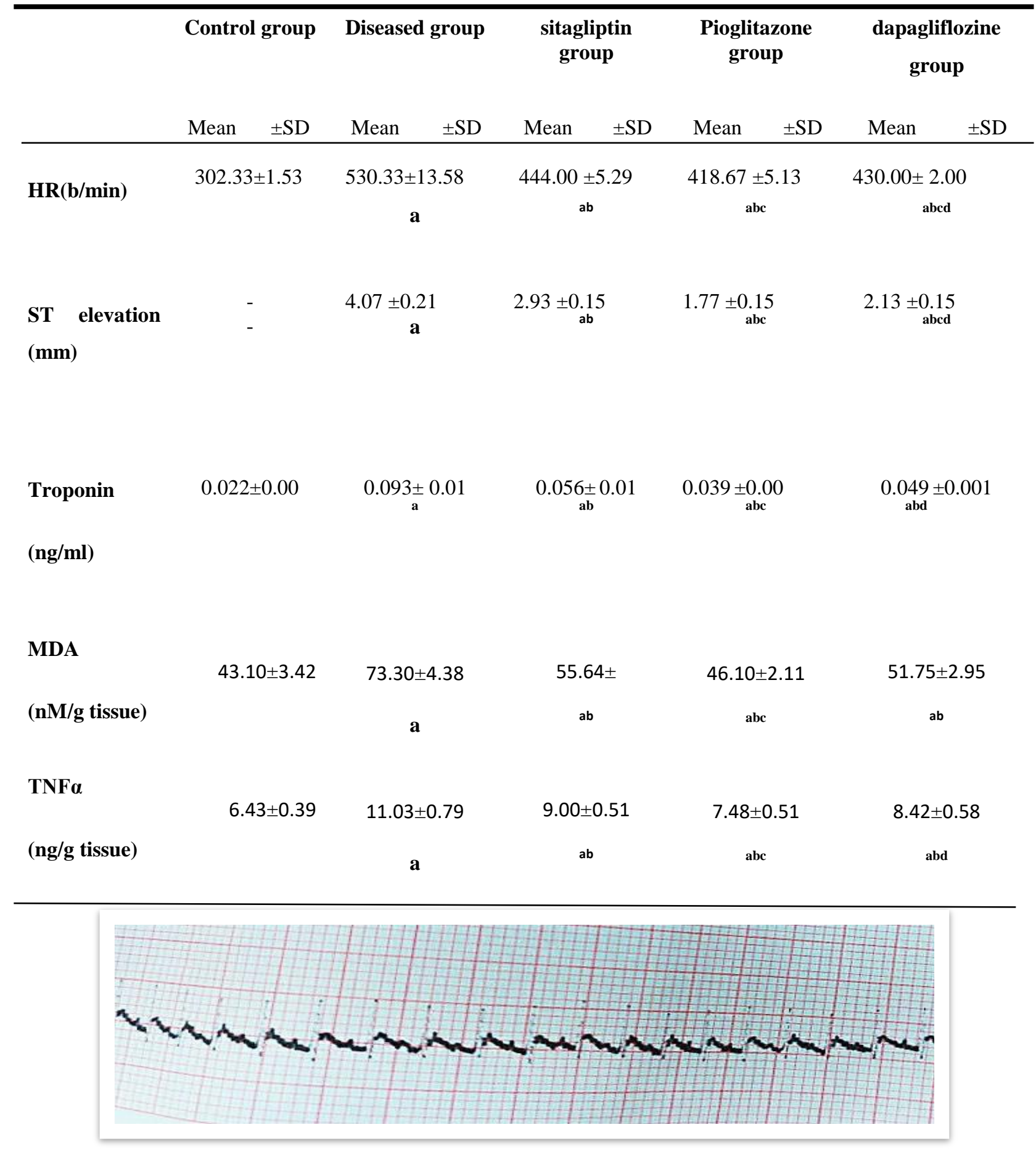

Figure. 1: ECG Tracing (lead II) of control normal rats. 


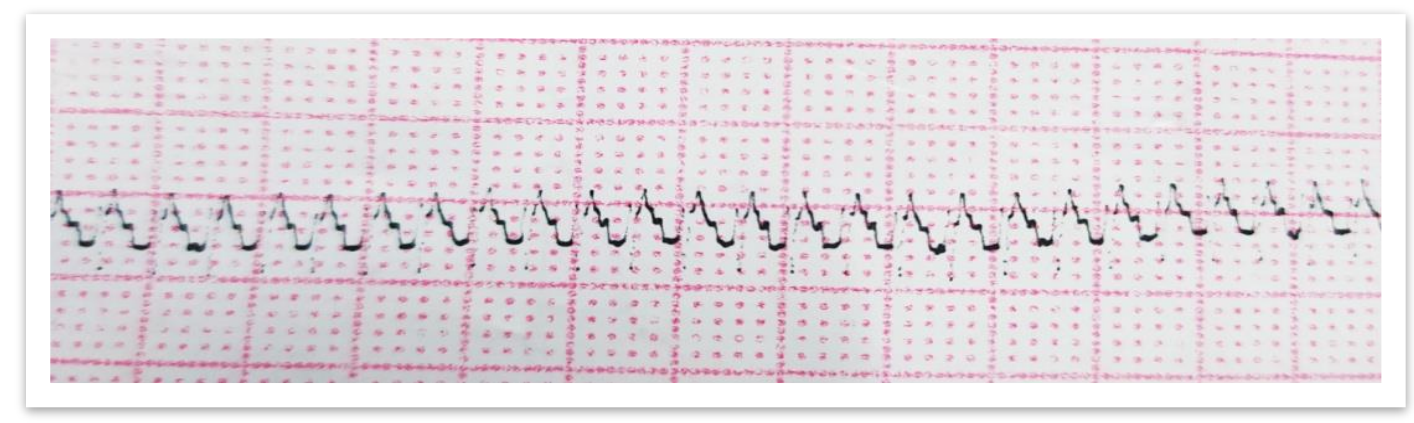

Figure 2: ECG Tracing (lead II) of infracted-diabetic non pre-treated rats.

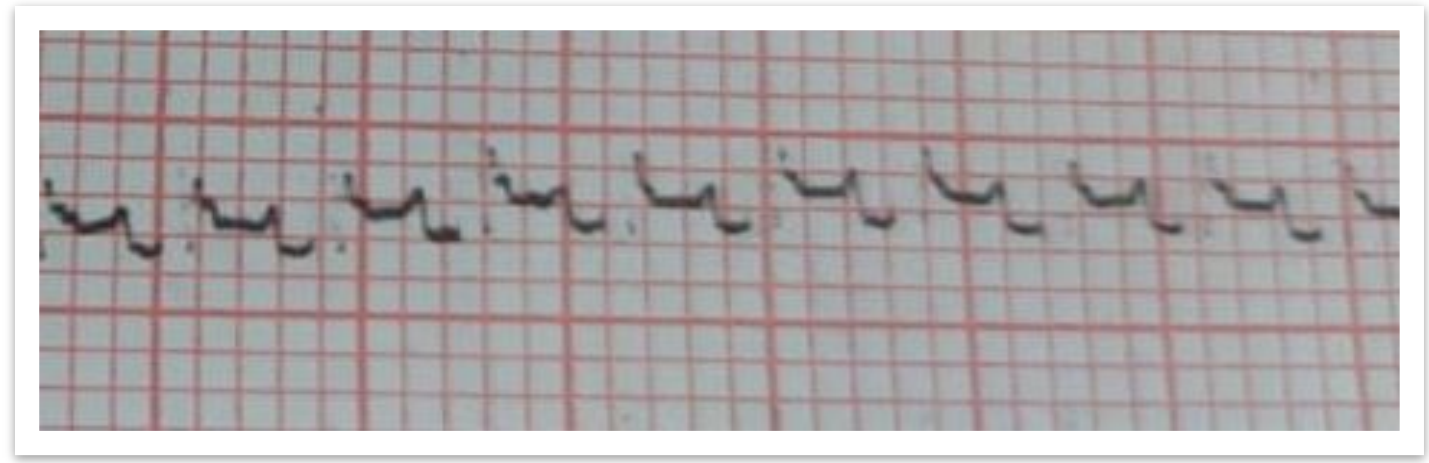

Figure 3: ECG Tracing (lead II) of infracted-diabetic sitagliptin pretreated rats.

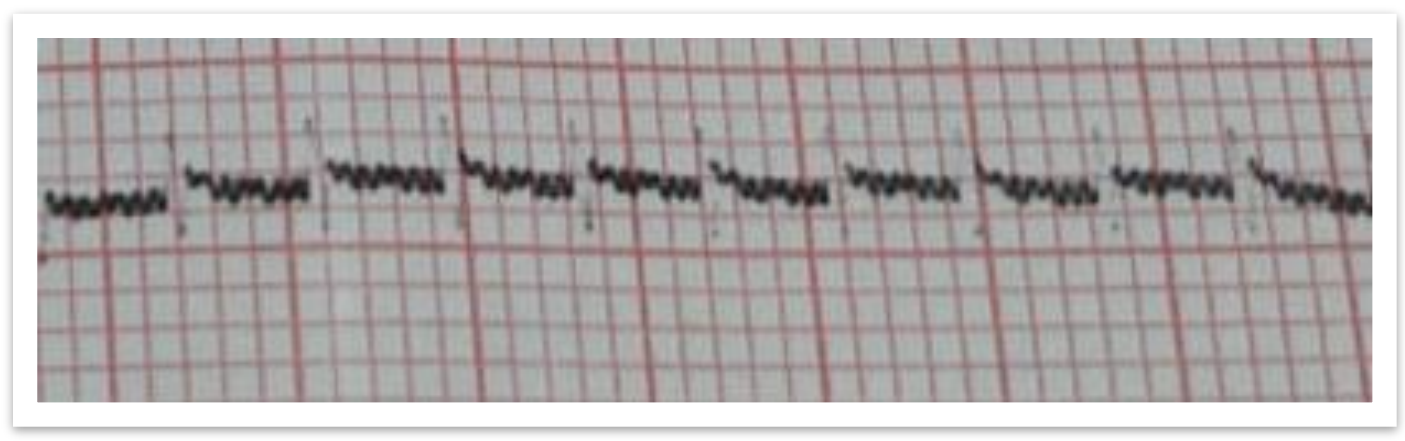

Figure 4: ECG Tracing (lead II) of infracted-diabetic pioglitazone pretreated rats.

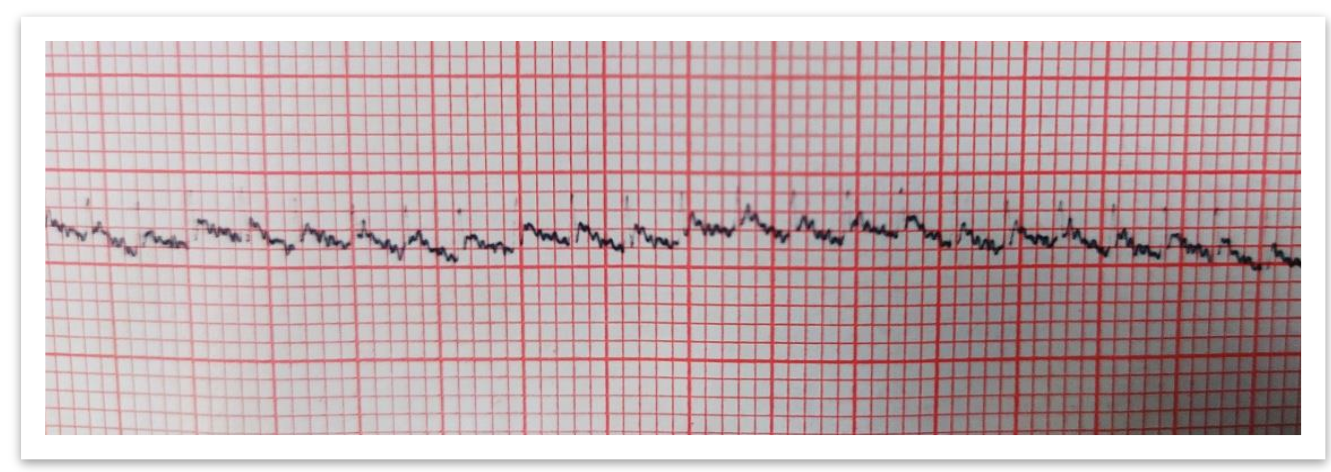

Figure 5: ECG Tracing (lead II) of infracted-diabetic dapagliflozine pretreated rats. 


\section{Histopathological changes:}
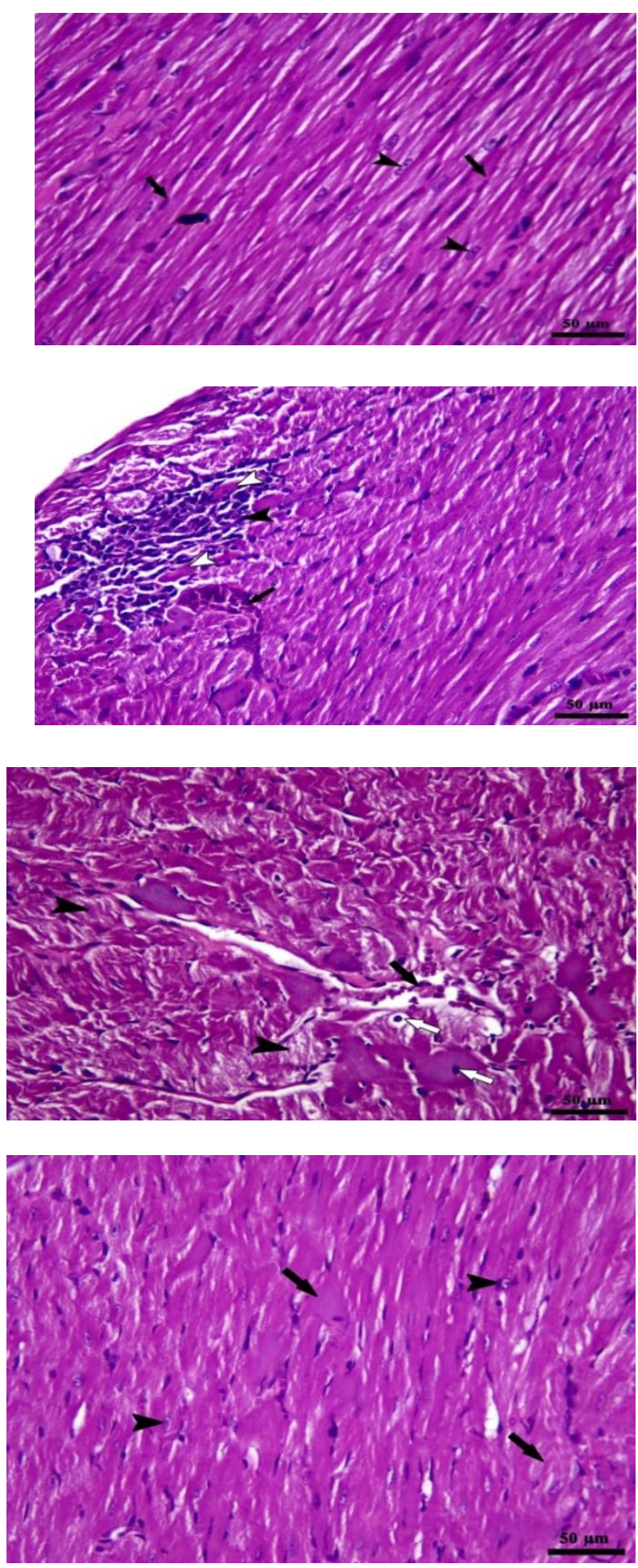

Fig. 6: photomicrograph of heart of G1 showing normal branched (arrow)and intact myocardial cells (arrow head).

$\mathrm{H} \& \mathrm{E}, \mathrm{bar}=50 \mu \mathrm{m}$

Fig. 7: photomicrograph of heart of G2 showing areas of focal necrosis of cardiomyocytes (white arrow head) surrounded by mononuclear cells (black arrow heads) and congested blood vessel (arrow).

$\mathrm{H} \& \mathrm{E}, \mathrm{bar}=50 \mu \mathrm{m}$

Fig. 8: photomicrograph of heart of G3 showing areas of degeneration and necrosis (arrow head) with pyknotic nuclei of cardiomyocytes (white arrow) and congestion of cardiac blood vessels (black arrow). H\&E, bar $=50 \mu \mathrm{m}$.

Fig. 9: photomicrograph of heart of G4 showing small area of degeneration of myocardial cells (arrow) in between intact myocardial cells (arrow heads).

$\mathrm{H} \& \mathrm{E}, \mathrm{bar}=50 \mu \mathrm{m}$. 


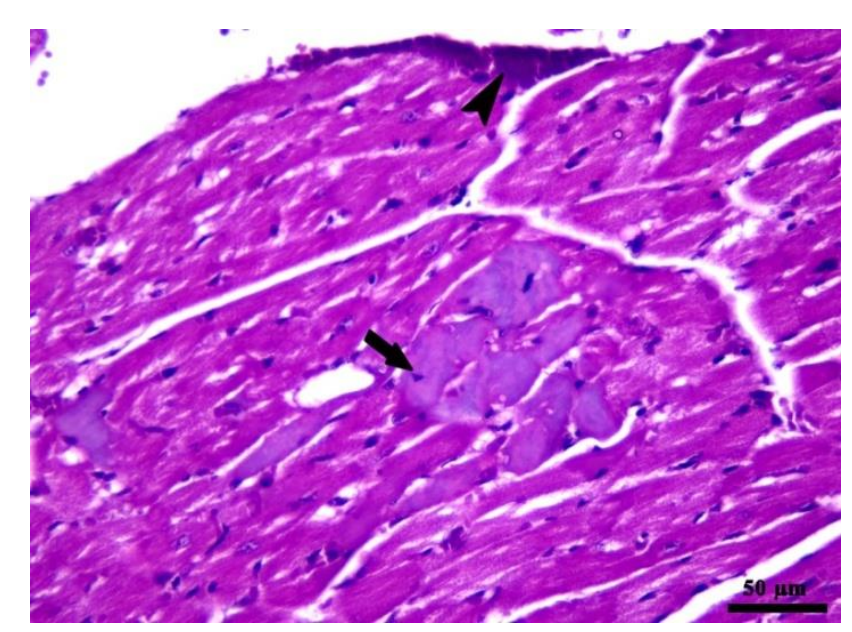

\section{Discussion}

The present study was designed to evaluate the effect of Sitagliptin, Pioglitazone and Dapagliflozine on experimentally-induced diabetes in rats regarding to their effect on blood glucose, lipid profile and kidney function. Also to detect the effect of Sitagliptin, Pioglitazone and Dapagliflozine on experimentally-induced myocardial infarction in diabetic rats regarding to their effect on heart rate, ST segment elevation, troponin level, TNFa, MDA and histopathology of the myocardium.

In the current work T2D was achieved by administration of $20 \%$ fructose solution in drinking water for 2 weeks, then intra peritoneal injection of a low dose STZ (40 $\mathrm{mg} / \mathrm{kg}$ b.w.) was attained [15\&16] .

Induction of diabetes in rats by fructose and STZ resulted in significant increases in
Fig. 10: photomicrograph of heart of G5 showing focal area of necrosis of cardiomyocytes (arrows) and congestion of blood vessels (arrow head). H\&E, bar $=50 \mu \mathrm{m}$. blood glucose, urea, creatinine, TC, LDL, triglyceride and significant decrease in HDL.

These finding are in agreement with several studies [33, 34, 35] which reported that HFSTZ-induced T2D is associated with significant increases in blood glucose, urea, creatinine, TC, LDL, triglyceride and significant decrease in HDL.

According to a certain study [16], MDA, a metabolite of lipid peroxidation, used to score oxidative stress in diabetic rats was also elevated. This may explain why treatment with HF and STZ may induce T2D through various mechanisms including IR and deterioration in $\beta$-cell functions .

Treatment with sitagliptin $10 \mathrm{mg} / \mathrm{kg} /$ day orally, pioglitazone $10 \mathrm{mg} / \mathrm{kg} / \mathrm{day}$ orally or dapagliflozine $1 \mathrm{mg} / \mathrm{kg} /$ day orally for 4 
weeks into diabetic rats resulted in significant improvement of blood glucose level, urea, creatinine, TC, LDL, triglyceride and significant increase in HDL level.

Regarding the effect of our drugs on blood glucose, dapagliflozine produced more decrease in blood glucose than other drugs. In support to our findings, the study [36] that discussed the efficacy of dapagliflozine versus sitagliptin on cardio-metabolic risk factors in Japanese patients with type 2 diabetes, reported that dapagliflozine was superior to sitagliptin regarding several secondary endpoints that modulate cardiometabolic risk, namely reducing fasting plasma glucose, insulin, uric acid, increasing high-density lipoprotein cholesterol, and suppressing the increase in serum creatinine and the decrease in estimated glomerular filtration rate. While another study [37] reported that sitagliptin treatment resulted in greater improvement in glycaemic control compared with dapagliflozine and was generally well tolerated.

Also, another study [38] reported that pioglitazone and sitagliptin achieved similar improvements in overall glycemic control in patients with type 2 diabetes inadequately controlled with metformin and a sulfonylurea. However there were some differences in terms of FPG, high sensitive C-reactive protein, lipids, body weight change and adverse events.

These data are in agreement with the study [39] of the cardiovascular efficacy of sitagliptin in patients with diabetes at high risk of cardiovascular disease which reported that sitagliptin showed a decrease in blood pressure associated with an improvement in albuminuria in addition to glycemic control.

In the study [40] which evaluated the effect of adding pioglitazone to T2DM patients' treatment protocol showed significant improvement in FBS and HbAlc as well as triglyceride and ALT levels. The consistent decrease of blood glucose and $\mathrm{HbA1C}$ indicates the efficacy of pioglitazone in controlling glycaemia.

The previous data were in agreement with the study [41] of the effect of dapagliflozine on the progression of the renal and liver fibrosis associated with type 2 diabetes, which reported that dapagliflozine reduced plasma glucose levels in addition to reduction of oxidative stress markers and inflammatory markers. Regarding to lipid profile, there was no significant difference between pioglitazone 
and dapagliflozine treated groups in all parameters of lipid profile. Moreover, there was no significant difference between them and the control group regarding to their effect on TG and HDL .Also, their effect on lipid profile was superior to the effect of sitagliptin.

The effect of our drugs on lipid profile were consistent with the study[42] that reported that dapagliflozine suppressed LDL-C and increased HDL2-C, but these findings were not observed after treatment with DPP-4 inhibitor sitagliptin.

In the study [43] which noticed the effects of DPP-4 inhibitor and SGLT2 inhibitor on lipid profile in patients with type 2 diabetes, it reported that SGLT2 inhibitor therapy shows a significant increase in HDL-C compared with the DPP-4 inhibitor.

Another study [44] reported that regarding fasting lipid levels, both sitagliptin and pioglitazone had a similar impact on each of the parameters, with an increase in HDL$\mathrm{C}$ and a decrease in the triglyceride levels. However, the extent to which HDL-C levels and triglyceride levels were affected favorably with pioglitazone was greater than that with sitagliptin.

These data are in agreement with the study [45] that detected the effect of sitagliptin on lipid profile in patients with type 2 diabetes mellitus, and reported that sitagliptin caused a significant decrease of TC, LDL$\mathrm{C}$ and non-HDL-C, particularly in patients with high baseline TG levels and those using strong statins. Another study [46] reported that treatment with pioglitazone was associated with great beneficial effects on blood lipid profile with a reduction in TGs level, a reduction in $\mathrm{TC}$, an increase in HDL, and a reduction in LDL-C .Also there is another study [47] reported that dapagliflozine has efficacy on increasing HDL-C levels and decreasing TG,TC and LDL-C levels. Regarding to kidney function, there was no significant difference between the three drugs on serum urea, but pioglitazone was superior in decreasing serum creatinine.

The results of kidney function are in agreement with the study [48] which reported that administration of sitagliptin showed a significant decrease in urea, BUN, creatinine and total protein versus diabetic group.

However, the study [49] of the long-term effects of sitagliptin in patients with type 2 diabetes mellitus and hypertension, reported that there was no evidence that treatment with sitagliptin can improve 
BUN or SCR in patients with type 2 diabetes mellitus and hypertension.

The study of the effects of pioglitazone on gentamicin-induced nephrotoxicity, reported that administration of medium dose of pioglitazone $(1 \mathrm{mg} / \mathrm{kg})$ with gentamicin showed a significant lower levels of serum urea and creatinine [50]. However, another study [51] reported that pioglitazone therapy had no effects on changes in mean values of serum creatinine, GFR\& BUN.

Another study [52] reported that, treatment with dapagliflozine produced significant reduction in creatinine clearance and blood urea nitrogen (BUN). However, the study [41] on the effect of Dapagliflozine on the progression of the renal and liver fibrosis associated with type 2 diabetes, reported that treatment with dapagliflozine had no effect on plasma creatinine levels.

The data of the present work revealed that, with respect to the control group, induction of acute myocardial infarction by isoprenaline in diabetic rats resulted in significant increases in heart rate, ST segment elevation and serum troponin level. These data are in agreement with several studies. [53 \& 54]. Also there was a significant increase in the level of $\mathrm{TNF} \alpha$ and
MDA this is in agreement with the previous studies. [53 \& 54].

ECG monitoring of isoprenaline-injected rats showed positive $\mathrm{T}$ wave and $\mathrm{ST}$ segment elevation that reflect the isoprenaline-induced myocardial ischemia and infarction. ECG pattern alterations by isoprenaline are in agreement with the previous studies. [53 \& 54].

Histopathological examination of cardiac tissue in the ISO-injected group revealed significant increase in histopathological cardiac injury score when compared to the control group, with evident dilatation in coronary blood vessel with thrombus, area of necrosis, severe hydropic degeneration and inflammatory cellular infiltrate with interstitial edema. These data are in agreement with the study [55] which reported that ISO-induced MI contributed to cause inflammatory cellular infiltrate with interstitial edema led to degeneration of cardiac myocytes and dilatation of blood vessels with thrombus formation. These observations along with biochemical changes in cardiac enzymes and cytokines confirm the severity of myocardial injury.

Our research revealed that sitagliptin supplementation $10 \mathrm{mg} / \mathrm{kg} / \mathrm{day}$ orally, pioglitazone $10 \mathrm{mg} / \mathrm{kg} /$ day orally or 
dapagliflozine $1 \mathrm{mg} / \mathrm{kg} /$ day orally for 4 weeks, before induction of MI restored the near normal ECG pattern with significant decrease in troponin levels, MDA and TNF $\alpha$ when compared to the MI group. Additionally, this was associated with significant decrease in cardiac histopathological injury score when compared to the MI group with improvement of histopathological finding in the form of mild hydropic degeneration without necrotic changes or cellular infiltration. Pioglitazone produced more improvement in troponin level, ECG changes and decreasing the infarct size, and so improving MDA and TNF $\alpha$ levels, followed by dapagliflozine, then sitagliptin. These findings are in agreement with several studies [56 \& 57] which reported that treatment by sitagliptin before induction of MI significantly decrease the infarct size, ST segment height and the elevated cardiac enzymes. Supporting to our findings, another study [58] reported that in patients with type 2 diabetes and coronary artery disease, treatment with sitagliptin resulted in a significantly lower rate of progression of coronary atherosclerosis compared with conventional treatment. While, another study [39] reported that there are no beneficial effects of sitagliptin on cardiac and endothelial function or on the levels of serum B-type natriuretic peptide and high-sensitive troponin $\mathrm{T}$.

\section{Conclusion}

Our study revealed that dapagliflozine produced more improvement in blood glucose level, and there was no significant difference between it and pioglitazone in improving lipid profile. Pioglitazone was superior in decreasing creatinine level, serum troponin, HR, ST elevation, MDA, TNF $\alpha$ and histopathological changes of myocardium. There was no significant difference between the three drugs regarding their effect on blood urea. Our drugs, mainly pioglitazone, may have a prophylactic effect against MI in diabetic rats. This may be due to their antioxidant and anti-inflammatory effect through reduction of MDA and TNF $\alpha$ respectively, glycemic control and improvement of dyslipidemia.

\section{References}

1. American Diabetes Association (2014): Diagnosis and classification of diabetes mellitus. Diabetes Care 37(Suppl. 1):S81-90. 10.2337/dc14-S081

2.Thygesen K., Alpert J., Jaffe A (2018): Fourth universal definition of myocardial infarction (2018) European Heart Journal. 2019;40(3):237-269.

3.Rosengren A, Edqvist J, Rawshani A, Sattar N, Franzen S, Adiels M,et al., ( 2018): Excess risk of 
hospitalisation for heart failure among people with type 2 diabetes. Diabetologia. 2018;61:2300-2309.

4.Holman R, Paul S, Bethel M, Matthews D, Neil $\mathrm{H}$ (2008): 10-year follow-up of intensive glucose control in type 2 diabetes. $N$ Engl J Med. 2008;359:1577-1589.

5.Rawshani A, Rawshani A, Franzén S, Eliasson B, Svensson A, Miftaraj M(2017): Mortality and cardiovascular disease in type 1 and type 2 diabetes. N Engl J Med. 2017;376:1407-1418.

6.Shi S., Koya D., Kanasaki K(2016): Dipeptidyl peptidase-4 and kidney fibrosis in diabetes. Fibrogenesis \& Tissue Repair. 2016;9:p. 1.

7.Scheen A (2010): Dipeptidylpeptidase-4 inhibitors (gliptins): focus on drug-drug interactions. Clin Pharmacokinet 2010; 49: 573-88.

8.Matsubara J, Sugiyama S, Sugamura K, Nakamura T, Fujiwara Y, Akiyama E(2012): A dipeptidyl peptidase-4 inhibitor, des-fluoro-sitagliptin, improves endothelial function and reduces atherosclerotic lesion formation in apolipoprotein Edeficient mice. J Am Coll Cardiol 2012; 59: 265-76.

9.Engel S, Round E., Golm G, Kaufman K, Goldstein B(2013):Safety and tolerability of sitagliptin in type 2 diabetes: pooled analysis of 25 clinical studies. Diabetes Therapy. 2013;4(1):119-145.

10.Kernan W, Viscoli C, Furie (2016): Pioglitazone after ischemic stroke or transient ischemic attack. N Engl J Med 2016;374:1321-31.

11.Motoki T, Kurobe H, Hirata $\mathrm{Y}$, Nakayama T, Kinoshita H, Rocco KA(2015). PPAR- $\gamma$ agonist attenuates inflammation in aortic aneurysm patients. Gen Thorac Cardiovasc Surg. 63:565-571. doi: 10.1007/s11748-015-0576-1.

12.Meng W, Ellsworth B, Nirschl A(2008): Discovery of dapagliflozin: a potent, selective renal sodiumdependent glucose cotransporter 2 (SGLT2) inhibitor for the treatment of type 2 diabetes. Med Chem. 2008;251:1145-9.

13. Inzucchi S, Zinman B, Wanner C, Ferrari R, Fitchett D, Hantel S,et al.,(2015): SGLT-2 inhibitors and cardiovascular risk: proposed pathways and review of ongoing outcome trials. Diab Vasc Dis Res. 2015;12(2):90-100.
14. Vennila L., Pugalendi K.V.,( 2010). Protective effect of sesamol against myocardial infarction caused by isoproterenol in Wistar rats. Redox Rep 15(1), 36-42.

15. Wilson, R. D., \& Islam, M. S. (2012): Fructose-fed streptozotocin-injected rat: an alternative model for type 2 diabetes. Pharmacological Reports, 64(1), 129-139.

16. Okolie, A. C., Kale, O. E., \& Osilesi, O. (2019): Chemoprotective effects of butanol fraction of Buchholzia coriacea (Capparidaceae) against type 2 diabetes and oxidative stress in male Wistar rats. Bioscience reports, 39(2).

17. Reimer R, Grover G, Koetzner L (2014): Combining sitagliptin/metformin with a functional fiber delays diabetes progression in Zucker rats. $\mathbf{J}$ Endocrinol. 2014;220:361-373.

18. Elrashidy, R.A.; Asker, M.E. and Mohamed, H.E. (2012): Pioglitazone attenuates cardiac fibrosis and hypertrophy in a rat model of diabetic nephropathy. J Cardiovasc Pharmacol Ther.17(3):324-333.

19. Tanaka A\& Node $K(2017)$ : Emerging roles of sodium-glucose cotransporter 2 inhibitors in cardiology. J Cardiol. 2017;69:501-7.

20. Nirmala, C. and Puvanakrishnan, R. (1994): Isoproterenol Induced myocardial infarction in rats: functional and biochemical alteration.

Med. Sci. Res.,22:575-7.

21. Date, Y., Nakazato, M., Murakami, N., Kojima, M., Kangawa, K., \& Matsukura, S. (2001):Ghrelin acts in the central nervous system to stimulate gastric acid secretion. Biochemical and biophysical research communications, 280(3), 904-907.

22. Burrin, J. M. and Price, C. P. (1985): Measurement of blood glucose. Ann. Clin. Biochem. 22: 327-342.

23. Bodor,G ;(1994):Cardiactroponin-I: a highly specific biochemical marker for myocardial infarction. J.Clin. Immunoassay.17: 40 -45.

24. Ohkawa, H., Ohishi, N., \& Yagi, K. (1979): Assay for lipid peroxides in animal tissues by 
thiobarbituric acid reaction. Analytical biochemistry, 95(2), 351-358.

25. Kasumagic-Halilovic, E.; Prohic, A. and Cavaljuga, S. (2011): Tumor necrosis factor-alpha in patients with alopecia areata. Indian Journal of Dermatology, 56(5):494-6.

26. Siedel, J.H.; Schlumberger, S.; Klose, J. and Washlefeld, A.W. (1991): J. Clin. Chem. Clin. Bioch; 19: 838. quoted from Boehringer Mannheim GMBH Diagnostic Manual.

27. Fossati,P.(1982): Enzymatic determination of serum triglycerides. Principle Clin. Chem; 28:2077.

28. Lopes-Virella, M.F.;Stone, P.; Ellis, S. and Colwell, J.A. (1977):Cholesterol determination in high-density lipoproteins separated by three different methods. Clin. chem; 23 (5):882-4.

29. Friedewald,W.T.;Levy,R.I. andFredrickson, D.S. (1972): Estimation of the concentration of lowdensity lipoprotein cholesterol in plasma, without use of the preparative ultracentrifuge. Clin. Chem; 18: 499-512.

30. Kaplan, A. (1984): Urea. Kaplan A et al. Clin Chem the C.V Mosby Co.St Louis Toronto Princeton. 1257-1260 and 437 and 418.

31. Murray, R.L.. (1984):creatinin.Kaplan A et al. Clin Chem The C.V Mosby Co.St Louis Toronto Princeton. 1261-1266 and 418.

32. Fischer, A. H., Jacobson, K. A., Rose, J., \& Zeller, R. (2008). Hematoxylin and eosin staining of tissue and cell sections. Cold Spring Harbor Protocols, 2008(5), pdb-prot4986.

33. Wilson, R. D., \& Islam, M. S. (2012): Fructose-fed streptozotocin-injected rat: an alternative model for type 2 diabetes. Pharmacological Reports, 64(1), 129-139.

34. Patel, R., Shah, P., Deshpande, S(2015):Fructose diet and low dose streptozotocin treatment induces the development of diabetic nephropathy in rats. Orient Pharm Exp Med 15, 305-312 (2015).
35. Kale, O.E., Akinpelu, O.B., Bakare, A.A. (2018). Five traditional Nigerian Polyherbal remedies protect against high fructose fed, Streptozotocin-induced type 2 diabetes in male Wistar rats. BMC Complement Altern Med 18, 160.

36. Fuchigami, A., Shigiyama, F., Kitazawa, T(2020): Efficacy of dapagliflozin versus sitagliptin on cardiometabolic risk factors in Japanese patients with type 2 diabetes: a prospective, randomized study (DIVERSITY-CVR). Cardiovasc Diabetol 19, 1 (2020).

37. Scott, R., Morgan, J., Zimmer, Z., Lam, R. L., O'Neill, E. A., Kaufman, K. D, et al., (2018). A randomized clinical trial of the efficacy and safety of sitagliptin compared with dapagliflozin in patients with type 2 diabetes mellitus and mild renal insufficiency: The CompoSIT-R study. Diabetes, Obesity and Metabolism, 20(12), 2876-2884.

38. Liu, Sung-Chen \& Chien, Kuo-Liong \& Wang, Chao-Hung, et al.,(2013). Efficacy and Safety of Adding Pioglitazone or Sitagliptin to Patients with Type 2 Diabetes Insufficiently Controlled with Metformin and a Sulfonylurea. Endocrine practice : official journal of the American College of Endocrinology and the American Association of Clinical Endocrinologists. 19. 1-30. 10.4158/EP13148.

39. Nakamura, T., Iwanaga, Y., Miyaji, Y(2016): Cardiovascular efficacy of sitagliptin in patients with diabetes at high risk of cardiovascular disease: a 12-month follow-up. Cardiovasc Diabetol 15, 54 (2016).

40. Aghamohammadzadeh, N., Niafar, M., Dalir Abdolahinia, E., Najafipour, F., Mohamadzadeh Gharebaghi, S., Adabi, K, et al., (2015): The effect of pioglitazone on weight, lipid profile and liver enzymes in type 2 diabetic patients. Therapeutic advances in endocrinology and metabolism, 6(2), $56-60$.

41. Tang, L., Wu, Y., Tian, M., Sjöström, C. D., Johansson, U., Peng, X. R., et al., (2017): Dapagliflozin slows the progression of the renal and liver fibrosis associated with type 2 
diabetes. American Journal of PhysiologyEndocrinology and Metabolism, 313(5), E563-E576.

42. Hayashi, T., Fukui, T., Nakanishi, N., Yamamoto, S., Tomoyasu, M., Osamura,et al.,(2017). Dapagliflozin decreases small dense low-density lipoprotein-cholesterol and increases high-density lipoprotein 2-cholesterol in patients with type 2 diabetes: comparison with sitagliptin. Cardiovascular diabetology, 16(1), 8.

43. Cha SA, Park YM, Yun JS(2017). A comparison of effects of DPP-4 inhibitor and SGLT2 inhibitor on lipid profile in patients with type 2 diabetes. Lipids in Health and Disease. 2017 Apr;16(1):58.

44. Chawla, S., Kaushik, N., Singh, N. P., Ghosh, R. K., \& Saxena, A. (2013). Effect of addition of either sitagliptin or pioglitazone in patients with uncontrolled type 2 diabetes mellitus on metformin: A randomized controlled trial. Journal of pharmacology \& pharmacotherapeutics, 4(1), 2732.

45. Shigematsu, E., Yamakawa, T., Kadonosono, K., \& Terauchi, Y. (2014): Effect of sitagliptin on lipid profile in patients with type 2 diabetes mellitus. Journal of clinical medicine research, 6(5), 327-335.

46. Filipova, E., Uzunova, K., Kalinov, K. (2017): Effects of pioglitazone therapy on blood parameters, weight and BMI: a meta-analysis. Diabetol Metab Syndr 9, 90.

47. Murat Calapkulu, Soner Cander, Ozen Oz Gul, Canan Ersoy,(2019).Lipid profile in type 2 diabetic patients with new dapagliflozin treatment;actualclinicalexperience data of six months retrospective lipid profile from single center,Diabetes \& Metabolic Syndrome: Clinical Research \& Reviews, Volume 13, Issue 2,2019,Pages 1031-1034,ISSN 1871-4021

\section{Mohamed M, Al-Eryan N, Elsayed F, Salah Eldin G} (2020): Effects of punica granatum peel extract and/ or sitagliptin on induced diabetic nephropathy in adult male albino rats. JRAM 2020; 1 (2): 104-115.
49. Ye, Z., Li, H., Lu, H., Su, Q., \& Li, L. (2017): Long-term effects of sitagliptin in patients with type 2 diabetes mellitus and hypertension: results from the PROLOGUE study. Oncotarget, 8(67), 111979111997.

50. Medić, B., Stojanović, M., Rovčanin, B(2019): Pioglitazone attenuates kidney injury in an experimental model of gentamicin-induced nephrotoxicity in rats. Sci Rep 9, 13689.

51. Shahidi, S., Pakzad, B., Mortazavi, M., Akbari, M., Seirafian, S., Atapour, A., et al., (2011): Reduction of proteinuria by pioglitazone in patients with nondiabetic renal disease. Journal of research in medical sciences : the official journal of Isfahan University of Medical Sciences, 16(11), 1459-1465.

52. Ali F. Abdel-Wahab, Ghazi A. Bamagous, Randa M. Al-Harizy, et al.,(2018).Renal protective effect of SGLT2 inhibitor dapagliflozin alone and in combination with irbesartan in a rat model of diabetic nephropathy,Biomedicine \& Pharmacotherapy,Volume 103,2018,Pages 5966,ISSN 0753-3322,

53. Zaafan, Mai \& Zaki, Hala \& El-Brairy, Amany ,et al., (2012): Isoprenaline-Induced Myocardial Infarction in Rats: Protective Effects of Hesperidin. Egyptian Journal of Basic and Clinical Pharmacology. 2. 10.11131/2012/101336.

54.Moradi-Arzeloo, M., Farshid, A. A., Tamaddonfard, E., \& Asri-Rezaei, S. (2016). Effects of histidine and vitamin $\mathrm{C}$ on isoproterenol-induced acute myocardial infarction in rats. Veterinary research forum : an international quarterly journal, 7(1), 4754.

55.Zaki, S. M., Abdalla, I. L., El Sadik, A. O., Mohamed, E. A., \&Kaooh, S.(2018):Protective Role of N-Acetylcysteine on Isoprenaline-Induced Myocardial Injury: Histological, Immunohistochemical and Morphometric Study. Cardiovascular toxicology, 18(1), 9-23

56. EL-sayed, Y., Fahmy, N., Khattab, M., Kabil, S., ElKashishy, K. (2019): Beneficial effects of sitagliptin ,metformin and their combination on myocardial ischemic and vascular changes in type- 
two diabetic rats. Zagazig University Medical Journal. Volume 27 - Issue 1.

57. Ibrahim, M. A., Geddawy, A., \& Abdel-Wahab, S. (2018): Sitagliptin prevents isoproterenol-induced myocardial infarction in rats by modulating nitric oxide synthase enzymes. European journal of pharmacology, 829, 63-69.
58. Li, B., Luo, Y. R., Tian, F., Chen, Y. D., Tian, J. W., Ding, Y., et al., (2020). Sitagliptin attenuates the progression of coronary atherosclerosis in patients with coronary disease and type 2 diabetes. Atherosclerosis. 2020 May;300:10-18.

To cite this article: Ahmed F. Bahriz, Yassmin M. Ismaiel, Abeer A. Abdelhameed, Fatma A. Elsayed. Effect of Sitagliptin, Pioglitazone and Dapagliflozine on Myocardial Infarction induced experimentally in diabetic rats. BMFJ 2021; 38 (academic issue): 147-165. DOI: 10.21608/bmfj.2021.58667.1373 